\title{
ESTUDO DAS COMUNIDADES DE ARANHAS (ARACHNIDA: ARANEAE) EM AMBIENTE DE MATA ATLÂNTICA NO PARQUE METROPOLITANO DE PITUAÇU - PMP, SALVADOR, BAHIA
}

\author{
Alder Oliveira-Alves, Marcelo Cesar Lima Peres, Marcelo Alves Dias, Gutemberg da Silva Cazais-Ferreira \& \\ Luciano Raimundo Alardo Souto \\ Biota Neotropica v5 (n1a) - http://www.biotaneotropica.org.br/v5n1a/pt/abstract?inventory+BN006051a2005
}

Recebido em 07/12/2003 - Aceito em 01/02/2005

Universidade Católica do Salvador, Instituto de Ciências Biológicas, Centro de Ecologia e Conservação Animal (ECOA),

Av. Prof ${ }^{\circ}$ Pinto de Aguiar, 2589, Pituaçu, 41740-090, Salvador, Bahia, Brasil. E-mail: alder oliveira@click21.com.br

\section{Abstract}

The Pituaçu Metropolitan Park (PMP) is a secondary Atlantic rainforest remnant with 425ha. New studies on ecology dynamics can help its management plan implementation and conservation. This paper analyses the border effect over the composition, diversity and richness of spider species in the PMP. The methods used were: nocturnal manual collection (CMN), and beating trays (GE). Twelve sampling units were randomly chosen ( 6 on the border and 6 on the interior) totalling an effort of 24 hours and 240 shrubs. Spider richness was estimated using Bootstrap, diversity using Shannon - Wiener (H). The similarity index of Jaccard was used to estimate the level of equality between the species composition in each unit. The statistical analysis were carried out using EstimateS@), Biostat 2.0@ and Diversity® packages. Comparisons betwenn spider richness were made using ANOVA. A total of 948 specimens were collected. Of these, 272 were adults, belonging to 80 species and 20 families. Bootstrap estimated 32 species for GE in the center and 34 on the border. Concerning CMN, estimates were of 45 species in the center and 53 on the border, verifying that for both sampling methods there was no significant difference between the areas (ANOVA p >0,05). In relation to diversity, Shannon-Wiener index found that for both sampling methods the diversity was higher on the border than in the center. These results show that there is a significant difference between the habitats located on the border and in the center. Jaccard similarity index resulted in $32,2 \%$ for CMN and 20,9\% for GE between the border and centre, indicating that there is a low level of similarity between the species composition of these areas. Among the dominant species in the center habitat, the orbweaving spiders were dominant, which was not observed on the remnant's border, where the runner spiders were dominant. The presence of a higher diversity of spider species on the border of the fragment is an unexpected result, since the majority of the studies indicate a higher diversity in the center, considering it a more preserved natural environment.

Key words: Spiders, Communities studies, Atlantic Forest, Board Effect, Bahia.

\section{Resumo}

O trabalho foi desenvolvido no Parque Metropolitano de Pituaçu (PMP), um fragmento de Mata Atlântica secundária com 425 ha, localizado nas coordenadas geográficas 1256’'S;38²4'W. Foi analisado o efeito de borda sobre a composição, diversidade e riqueza em espécies de aranhas do PMP. Foram utilizados dois métodos: coleta manual noturna (CMN) e guarda-chuva entomológico (GE). Foram escolhidos 12 pontos arbitrariamente, seis de borda e seis de centro, totalizando 24h e 240 arbustos. A riqueza foi estimada pelo índice de Bootstrap através do software EstimateS e a diversidade pelo índice de Shannon-Wiener (H). Foi aplicado o índice de similaridade de Jaccard para estimar o grau de semelhança entre a composição de espécies dos ambientes. As análises estatísticas foram feitas a partir do software Bioestat 2.0 e Diversity $\odot$, utilizando-se para a comparação da riqueza estimada entre os ambientes, o teste ANOVA: um critério. Foram coletados 948 espécimes, sendo 272 indivíduos adultos, agrupados em 20 famílias e 80 espécies. A Partir do índice de riqueza Bootstrap estimou-se para o método de coleta GE 32 espécies para o centro e 34 para a borda, quanto a CMN foi estimado 45 espécies para o centro e 53 para a borda, verificando-se que em ambos os métodos de amostragem não houve diferença significativa entre as áreas (ANOVA, p>0,05). Com relação à diversidade, observou-se que em ambos os métodos de coleta que o índice de diversidade de Shannon-Wiener foi maior na borda que no centro, apontando diferenças bastante significativas, entre os ambientes de borda e centro. O índice de similaridade de Jaccard em relação à composição de espécies entre borda e centro foi de 32,2\% para CMN e 20,9\% para GE apresentando assim um baixo grau de similaridade na composição dessas espécies entre as áreas. Dentre as espécies dominantes no ambiente de centro as aranhas construtoras de teia foram mais abundantes, fato este que não foi observado na borda do fragmento, ocorrendo predominância de aranhas errantes. A presença de maior diversidade de espécies de aranhas na borda do fragmento, apresentase como um resultado inusitado, devido às suas características físicas do centro serem mais conservadas. Desta forma infere-se em nossos resultados a atuação do efeito de borda como uma barreira natural, impedindo assim o fluxo de espécies entre os ambientes, contudo esse resultado também propõe que exista um fator mais expressivo atuando na diversidade de espécies de aranhas concentrando-as na borda do fragmento.

Palavras-chave: Aranha, Estudo de Comunidade, Mata Atlântica, Efeito de borda, Bahia 


\section{Introdução}

A alta velocidade de destruição de florestas tropicais, aliada a extinções de espécies, exige o desenvolvimento de estratégias de conservação e uso sustentado dos fragmentos remanescentes, bem como de recuperação de áreas degradadas (Espírito-Santo et al. 2002).

Fragmentos florestais são áreas de vegetações naturais interrompidas por barreiras antrópicas ou naturais, capazes de diminuir, significativamente, o fluxo de animais, pólen ou sementes (Viana 1992). Em se tratando de uma ação antrópica os fragmentos são advindos de vários anos de desmatamento e constituem hoje um dos maiores desafios para a conservação (Espírito-Santo et al. 2002).

A borda, o tipo de vizinhança, o grau de isolamento e o tamanho efetivo dos fragmentos florestais são os principais fatores que devem ser considerados, para medir as alterações dos processos biológicos de determinado ecossistema (Benedetti \& Zani Filho 1993), além disso, a predação, a competição e as interações mutualísticas também variam ao longo do mosaico de habitats e provavelmente também influenciam a estrutura das comunidades e a dinâmica das populações (Levey 1988, Hacker \& Gaines 1997), nesses fragmentos. Atualmente a Mata Atlântica está reduzida à apenas 5\% do original, sendo considerado um dos principais hotspot (Myers et al. 2000).

O Efeito de Borda (EB) é um fenômeno que ocorre no ecótone promovendo o aumento da densidade e riqueza em espécie (Odum 1988), no entanto, em ambientes fragmentados que sofrem intensa ação antrópica, pode ocorrer um efeito inverso ao proposto, verificando uma redução na densidade e riqueza em espécie, assim como uma alteração na estrutura e dinâmica das comunidades de plantas (Laurence et al. 1998). Desta forma, o EB possivelmente está entre um dos mais evidentes e significativos fatores que atuam sobre os fragmentos florestais.

Os artrópodes compreendem cerca de $75 \%$ dos animais existentes. Dentre os representantes deste grupo às aranhas compreendem uma significante porção da diversidade de artrópodes terrestres (Toti et al. 2000), ocupando a posição de consumidoras secundárias, sendo predadores generalistas em ecossistemas terrestres (Wise 1993; Foelix 1996; Breene et al. 1993). Devido à grande riqueza em espécies, 38.663 espécies (Platnick 2004) e à sensibilidade a diversos fatores ambientais, as aranhas formam um grupo taxonômico indicado para avaliar o estado de conservação de fragmentos florestais, visto que a estrutura do habitat pode influenciar bastante a composição e a riqueza das comunidades de aranhas de florestas tropicais (Uetz 1991, Santos 1999). Höfer \& Brescovit (1997) sugerem o uso das mesmas como bioindicadores para diagnóstico de uma determinada área.
Neste trabalho analisamos o efeito de borda sobre a composição, diversidade e riqueza em espécies de aranhas do PMP, visando avaliar como e em que nível o efeito de borda interfere direta e indiretamente sobre a comunidade de aranhas.

\section{Material e Métodos}

O Parque Metropolitano de Pituaçu (PMP), localizase no município de Salvador, compreendido entre as coordenadas geográficas $12^{\circ} 56^{\prime} \mathrm{S}$ e $38^{\circ} 24^{\prime} \mathrm{W}$ (Conceição et al. 1998). A Origem da lagoa de Pituaçu é de 1906 quando houve o represamento do Rio Pituaçu, sendo a partir de 1977, considerado uma Unidade de Conservação, sob o Decreto Municipal no 5.158. O Parque é uma das maiores unidades de conservação de Mata Atlântica dentro da área urbana da região Metropolitana do Salvador, compreendendo 425 ha de área conservada, com vegetação ombrófila densa e formações vegetais de restinga (Conceição et al. 1998), formando um ecótono (Teles \& Bautista 2001). Apesar de ser um fragmento remanescente secundário de Mata Atlântica, em estágio de regeneração inicial, médio e avançado (Teles \& Bautista 2001), sofre diversas ações antrópicas, como por exemplo, atividade de pesca clandestina, lançamento de esgotos na lagoa, destruição da mata ciliar, tráfego intenso de moradores e introdução de animais silvestres.

Foram utilizados dois métodos de coletas: guardachuva entomológico (GE) e coleta manual noturna (CMN), adaptados de Coddington et al. (1991) e empregados em 12 pontos amostrais escolhidos de forma arbitrária, e divididos em seis pontos de borda e seis pontos de centro (Figura 1). O esforço amostral foi de uma hora de CMN ida e volta, em um transecto de $30 \mathrm{x}$ cinco metros, e para o GE foram considerados 10 arbustos, como sendo uma unidade amostral dentro de cada ponto. Ambos realizados por dois pesquisadores que se revezaram para amostrar os 12 pontos, totalizando um esforço de $24 \mathrm{~h}$ e 240 arbustos. O material coletado, foi depositado na coleção científica do Centro de Ecologia e Conservação Animal - ECOA/ICB/UCSAL.

Além das variáveis de fauna, foram também avaliadas as variáveis que compõem a estrutura física do PMP: cobertura de herbácea, cobertura e profundidade da serrapilheira, circunferência a altura do peito - CAP de lenhosas, número e grau de decomposição de troncos caídos.

Foram analisadas as variáveis da estrutura física dos 12 pontos amostrais. Em cada ponto amostral, foi delimitado um transecto de $30 \mathrm{x}$ cinco metros, dividido em quatro parcelas de $25 \mathrm{~m}^{2}$ distadas $10 \mathrm{~m}$ uma da outra. Em cada parcela foram atribuídos valores para cobertura de herbácea e cobertura de serrapilheira (0-33\%, pouco; 34-66\%, médio; $67-100 \%$, muito); quantificados a profundidade média da serrapilheira; número de troncos caídos e o estado de conservação dos troncos (recente, intermediário e avançado), foram considerados troncos a partir de $10 \mathrm{~cm}$ de 


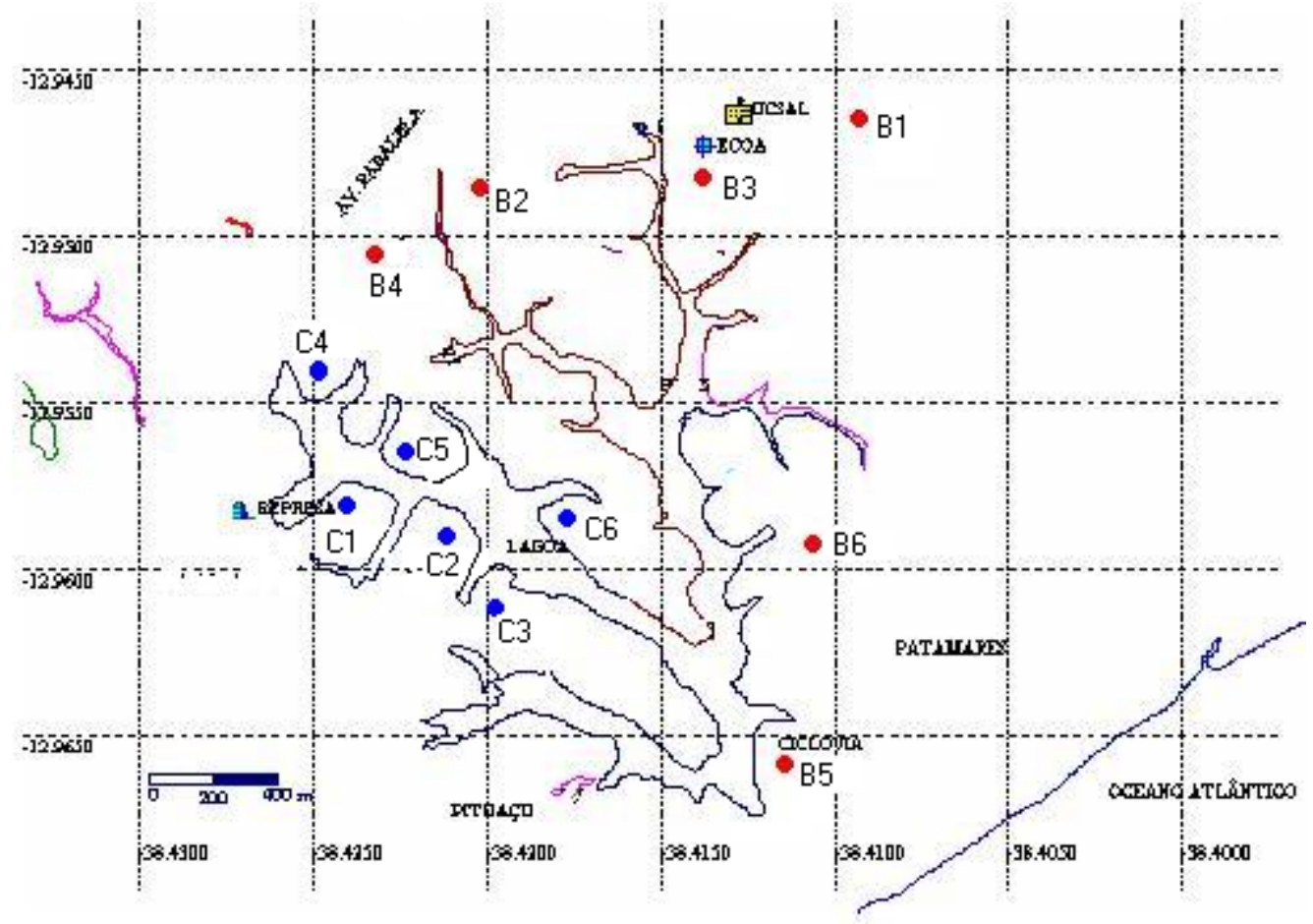

Figura 1: Mapa com a localização dos pontos de borda (B1 a B6

e centro C1 a C6

) no Parque Metropolitano de Pituaçu.

diâmetro; mensurados CAP a cerca de 1,5m do solo de todas as arvores e arbustos com diâmetro mínimo de quatro centímetros. Não foram mensurados CAP de palmeiras.

Análises estatísticas foram feitas a partir do software Bioestat 2.0 (Ayres et al. 2000) e Diversity (C) (Henderson \& Seaby 1998). Para a comparação da riqueza de espécies entre os ambientes (centro e borda) foi estimado o índice de riqueza de Bootstrap para os 12 pontos de amostras em ambos os métodos (CMN e GE), sendo aplicado o teste t, visto que o teste de Kolmogorov e Sminorv verificou que todas as amostras tinham distribuição normal $(p>0,10)$, para testar a diferença entre os ambientes. O índice de similaridade de Jaccard foi utilizado para avaliar o grau de semelhança da composição de espécies entre as áreas. A diversidade das espécies entre os ambientes de borda e centro foi avaliada pelo índice de Shannon-Wiener $(\mathrm{H})$. Para comparar as variáveis estruturais entre os ambientes foi utilizado o teste não paramétrico de Mann-Whitney - teste U, visto que o teste de Kolmogorov e Sminorv verificou que todas as amostras não tinham distribuição normal $(\mathrm{p}<0,001)$.

\section{Resultados e Discussão}

Foram coletadas 948 aranhas, sendo 272 adultas, agrupados em 20 famílias e 80 espécies, 56 espécies ocorreram na borda e 49 no centro (Tabela 1). Dentre os indivíduos adultos 133 (48,90\%) foram coletados na borda, desses 87
(65,40\%) através do método CMN e 46 (34,60\%) através do GE, no centro foram coletados 139 (51,10\%) sendo 93(66,90\%) na CMN e 46 (33,10\%) no GE. A partir do índice de riqueza Bootstrap estimou-se para o método de coleta GE 34 espécies para a borda e 32 para o centro, no entanto esta diferença não foi significativa ( $\mathrm{t}=0,4842$; $\mathrm{gl}=22$; $\mathrm{p}=0,6330$ ), para a $\mathrm{CMN}$ foi estimado 45 espécies para o centro e 53 para a borda, verificando-se também que não houve diferença significativa entre os ambientes $(\mathrm{t}=1,001 ; \mathrm{gl}=22 ; \mathrm{p}=0,3279)$. Em todas as estimativas de riqueza a curva de espécies observadas não alcançou a assíntota, indicando que a área apresenta uma riqueza maior do que a estimada (Figura 2). Com relação à diversidade, observou-se em ambos os métodos de coleta que o índice de diversidade de Shannon-Wiener foi maior na borda (CMN $H=3,3741$ e GE $H=3,0701$ ) que no centro (CMN $H=3,0844$ e GE $H=2,8705$ ), apontando diferenças bastante significativas, entre os ambientes de borda e centro na CMN ( $d=0,9628)$ e no GE ( $d=08354)$. O índice de similaridade de Jaccard em relação à composição de espécies entre borda e centro foi de 32,2\% para CMN e 20,9\% para GE, apresentando assim um baixo grau de similaridade na composição dessas espécies entre as áreas.

Quando borda e centro, foram comparadas no que diz respeito à estrutura física, apresentou diferenças significativas, com $\mathrm{p}=0,0145$ para cobertura de herbácea mais acentuada no centro, $\mathrm{p}=0,0035$ maior profundidade de serrapilheira e $\mathrm{p}=0,0136$ para valores mais significantes de 

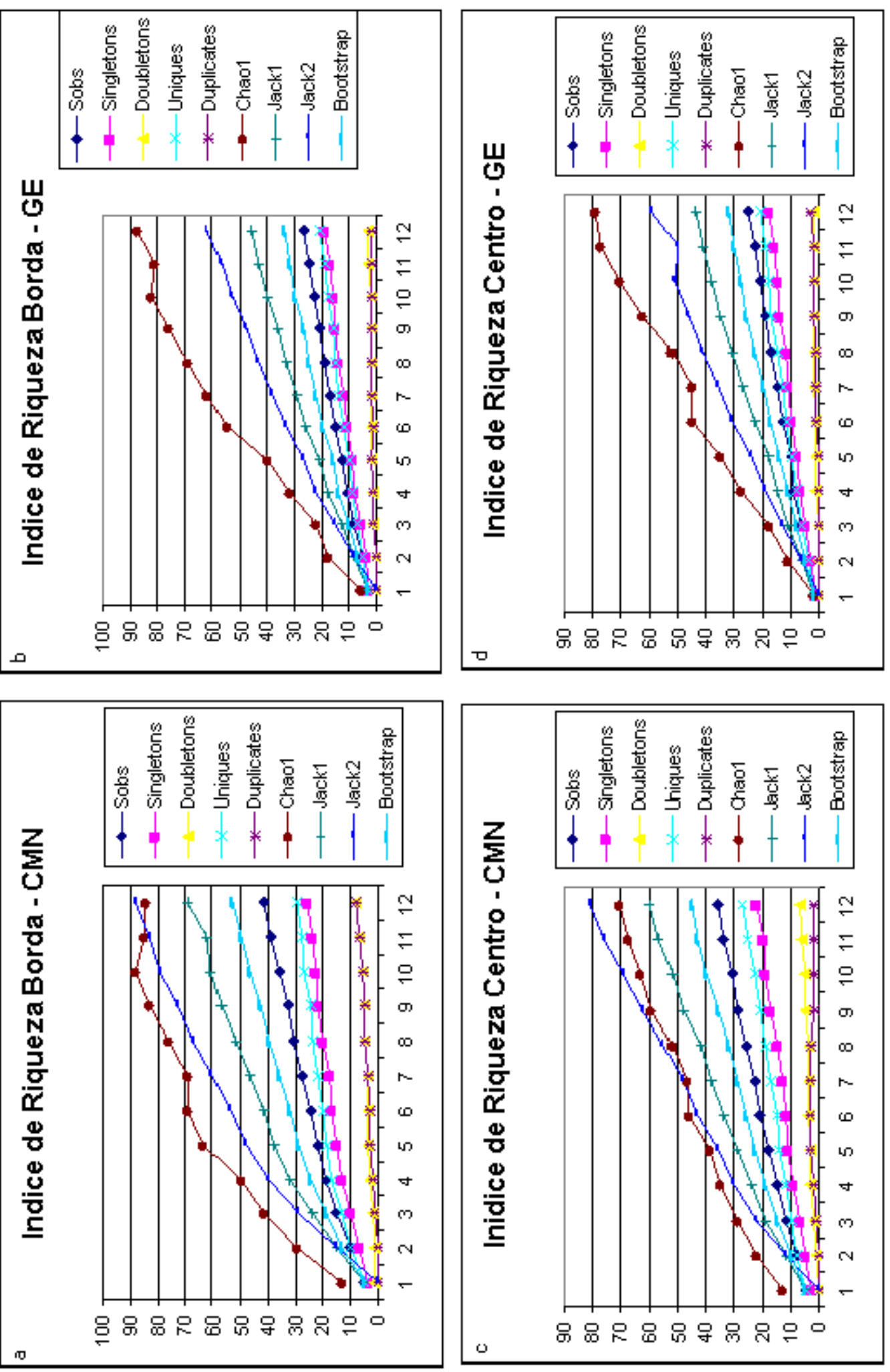
Tabela 1: Lista de espécies e número de adultos coletados em ambientes de borda e centro, em dois métodos de coleta: coleta manual noturna $(C M N)$ e guarda-chuva entomológico (GE) no Parque Metropolitano de Pituaçu (PMP) - Salvador - Bahia - Brasil.

\begin{tabular}{|c|c|c|c|c|c|c|}
\hline Família & Espécie ou Morfoespécie & GE - Borda & GE - Centro & CMN - Borda & CMN - Centro & Total \\
\hline \multirow[t]{5}{*}{ Anyphaenidae } & Wulfila sp. & 5 & 1 & 1 & 1 & 8 \\
\hline & Xiruana sp. & 0 & 0 & 1 & 0 & 1 \\
\hline & Anyphaenidae sp.1 & 0 & 0 & 2 & 0 & 2 \\
\hline & Anyphaenidae sp.2 & 0 & 1 & 0 & 1 & 2 \\
\hline & Anyphaenidae sp.3 & 1 & 0 & 0 & 0 & 1 \\
\hline \multirow[t]{18}{*}{ Araneidae } & Acacesia sp. & 0 & 0 & 0 & 2 & 2 \\
\hline & Alpaida delicata (Keyserling, 1892) & 0 & 1 & 0 & 0 & 1 \\
\hline & Araneus tijuca (Levi, 1988) & 0 & 0 & 3 & 1 & 4 \\
\hline & Argiope argentata (Fabricius, 1775) & 0 & 0 & 2 & 1 & 3 \\
\hline & Cyclosa sp. & 0 & 0 & 2 & 2 & 4 \\
\hline & Eriophora sp. & 1 & 1 & 6 & 2 & 10 \\
\hline & Eustala sp. & 0 & 1 & 1 & 1 & 3 \\
\hline & Micrathena fissispina (Strand, 1908) & 0 & 0 & 0 & 1 & 1 \\
\hline & Micrathena flaveola (Koch, C. L. 1839) & 0 & 0 & 0 & 1 & 1 \\
\hline & Micrathena triangularispinosa (De Geer, 1778) & 0 & 0 & 0 & 1 & 1 \\
\hline & Araneidae sp.1 & 0 & 0 & 1 & 2 & 3 \\
\hline & Araneidae sp.2 & 0 & 0 & 0 & 1 & 1 \\
\hline & Araneidae sp.3 & 1 & 0 & 1 & 1 & 3 \\
\hline & Araneidae sp.4 & 0 & 2 & 7 & 1 & 10 \\
\hline & Araneidae sp.5 & 0 & 0 & 2 & 0 & 2 \\
\hline & Araneidae sp.6 & 0 & 0 & 0 & 1 & 1 \\
\hline & Araneidae sp.7 & 1 & 0 & 0 & 0 & 1 \\
\hline & Araneidae sp. 8 & 0 & 0 & 2 & 0 & 2 \\
\hline Clubionidae & Elaver sp. & 0 & 0 & 1 & 0 & 1 \\
\hline Corinnidae & Coriniidae sp. & 0 & 0 & 0 & 1 & 1 \\
\hline Deinopidae & Deinopis sp. & 0 & 0 & 1 & 8 & 9 \\
\hline Lycosidae & Lycosa gr. nordeskijoldii & 0 & 0 & 0 & 2 & 2 \\
\hline \multirow[t]{2}{*}{ Mimetidae } & Gelanor sp.1 & 2 & 4 & 1 & 4 & 11 \\
\hline & Gelanor sp.2 & 1 & 0 & 0 & 0 & 1 \\
\hline \multirow[t]{2}{*}{ Oxyopidae } & Peucetia rubrolineata (Keyserling, 1877) & 0 & 0 & 3 & 0 & 3 \\
\hline & Peucetia sp. & 0 & 0 & 1 & 0 & 1 \\
\hline \multirow[t]{2}{*}{ Pholcidae } & Mesabolivar sp. & 0 & 10 & 13 & 15 & 38 \\
\hline & Pholcidae sp. & 0 & 5 & 0 & 7 & 12 \\
\hline Pisauridae & Architis sp. & 1 & 0 & 0 & 0 & 1 \\
\hline Salticidae & Lyssomanes sp.1 & 3 & 0 & 1 & 0 & 4 \\
\hline & Lyssomanes sp.2 & 1 & 0 & 0 & 0 & 1 \\
\hline & Thiodina sp. & 0 & 2 & 1 & 0 & 3 \\
\hline & Salticidae sp.1 & 5 & 3 & 0 & 0 & 8 \\
\hline & Salticidae sp.2 & 0 & 0 & 0 & 1 & 1 \\
\hline & Salticidae sp.3 & 0 & 0 & 1 & 0 & 1 \\
\hline & Salticidae sp.4 & 0 & 0 & 0 & 1 & 1 \\
\hline & Salticidae sp.5 & 0 & 1 & 0 & 0 & 1 \\
\hline & Salticidae sp.6 & 0 & 1 & 0 & 0 & 1 \\
\hline & Salticidae sp.7 & 1 & 0 & 3 & 1 & 5 \\
\hline & \begin{tabular}{|l|} 
Salticidae sp. 8 \\
\end{tabular} & 3 & 1 & 1 & 0 & 5 \\
\hline & Salticidae sp.9 & 1 & 0 & 0 & 0 & 1 \\
\hline & Salticidae sp.10 & 1 & 0 & 0 & 0 & 1 \\
\hline & Salticidae sp.11 & 1 & 0 & 0 & 0 & 1 \\
\hline & Salticidae sp.12 & 1 & 0 & 6 & 0 & 7 \\
\hline & Salticidae sp.13 & 0 & 0 & 1 & 0 & 1 \\
\hline & Salticidae sp.14 & 0 & 0 & 1 & 0 & 1 \\
\hline & Salticidae sp.15 & 0 & 0 & 1 & 0 & 1 \\
\hline Scytodidae & Scytodes fusca (Walckenaer, 1837) & 1 & 1 & 0 & 0 & 2 \\
\hline & Scytodidae sp.1 & 1 & 1 & 4 & 0 & 6 \\
\hline & Scytodidae sp.2 & 0 & 0 & 2 & 1 & 3 \\
\hline Sparassidae & Sparianthinae sp. & 0 & 0 & 1 & 0 & 1 \\
\hline & Sparassidae sp. & 5 & 2 & 1 & 1 & 9 \\
\hline Tetragnathidae & Chrysometa sp. & 0 & 0 & 0 & 1 & 1 \\
\hline & Leucauge aff. argyra (Walckenaer, 1842) & 2 & 1 & 2 & 11 & 16 \\
\hline & Nephila clavipes (Mello-Leitão, 1944b) & 0 & 0 & 1 & 8 & 9 \\
\hline & Tetragnathidae sp.1 & 0 & 0 & 1 & 0 & 1 \\
\hline & Tetragnathidae sp.2 & 0 & 0 & 0 & 2 & 2 \\
\hline & Tetragnathidae sp. 3 & 0 & 1 & 0 & 0 & 1 \\
\hline & Tetragnathidae sp.4 & 0 & 1 & 0 & 0 & 1 \\
\hline & Tetragnathidae sp.5 & 1 & 0 & 1 & 0 & 2 \\
\hline Theridiidae & Episinus sp. & 0 & 0 & 1 & 0 & 1 \\
\hline & Spintharus gracilis (Keyserling, 1886) & 1 & 0 & 0 & 0 & 1 \\
\hline & Tidarren sp. & 0 & 0 & 1 & 0 & 1 \\
\hline & Theridiidae sp.1 & 0 & 1 & 0 & 0 & 1 \\
\hline & Theridiidae sp.2 & 0 & 0 & 1 & 0 & 1 \\
\hline & Theridiidae sp.3 & 0 & 0 & 1 & 0 & 1 \\
\hline Thomisidae & Deltoclita sp. & 2 & 0 & 0 & 0 & 2 \\
\hline & Onoculus sp. & 1 & 0 & 0 & 0 & 1 \\
\hline & Tmarus sp.1 & 0 & 1 & 0 & 4 & 5 \\
\hline & Tmarus sp.2 & 1 & 0 & 2 & 1 & 4 \\
\hline & Thomisidae sp.1 & 0 & 1 & 0 & 1 & 2 \\
\hline & Thomisidae sp.2 & 0 & 1 & 0 & 0 & 1 \\
\hline & Thomisidae sp.3 & 0 & 1 & 0 & 0 & 1 \\
\hline & Thomisidae sp.4 & 1 & 0 & 0 & 2 & 3 \\
\hline Uloboridae & Uloboridae sp. & 0 & 0 & 1 & 1 & 2 \\
\hline Total & & 41 & 45 & 85 & 92 & 272 \\
\hline
\end{tabular}


CAP para a borda do parque. Valores estatisticamente não significantes foram apresentados ao avaliar cobertura de serrapilheira, número e grau de decomposição de troncos caídos com p > 0,05, Mann-Whitney.

Estruturalmente o PMP caracteriza-se por ser uma área de Mata Atlântica secundária, com setores antropizados, contudo, guardando ainda muitos aspectos da sua caracterização primitiva de ecótono, apresentando assim, um grande potencial biológico (Teles \& Bautista 2001). Cruz Rios et al (2003) ao analisar as variáveis ambientais como temperatura do ar (A_Tar), temperatura do solo (A_Tsol) e umidade relativa do ar (A_Urar) observaram que tais constantes ambientais indicaram diferenças ao longo dos pontos amostrais. Os valores de A_Urar apresentaram diferença significativa entre as áreas e também quando comparadas com as demais medidas. As freqüências de CAP (Circunferência ao nível do Peito), realizadas em vegetais e hábito arbóreo, indicaram que existe uma diferença muito significativa entre os pontos de centro e borda, fato que corroboraria a teoria do efeito de borda em si comprovando uma variação nas comunidades ali representadas.

Ao comparar as comunidades de aranhas presentes no PMP, verificou-se que as famílias estão distribuídas de forma distinta entre borda e centro. Classificando as em guildas de acordo com o proposto por Höfer \& Brescovit (2001) para aranhas da Amazônia, observa-se o seguinte resultado:

- aranhas caçadoras, predominantes na borda: NAA, Anyphaenidae com três espécies exclusivas e Sparassidae com uma; NAR, Scytodidae com duas espécies exclusivas, Clubionidae e Mimetidae com uma espécie exclusiva, Salticidae com dez e Thomisidae com três; DAH, Oxyopidae com duas espécies exclusivas e DGN, Coriniidae com uma;

- aranhas construtoras de teias predominantes no centro: AOW, Araneidae com oito espécies exclusivas, Tetragnathidae com três e Uloboridae; SSW, Lycosidae, Pholcidae e Pisauridae com uma espécie exclusiva cada; ASWB, Theridiidae com uma espécie exclusiva; NGW, Deinopidae com uma.

As espécies que apresentaram um número de ocorrência igual ou superior a cinco espécimes foram consideradas dominantes, representando 61,80\% da amostragem de adultos. Dentre as espécies dominantes, as aranhas construtoras de teia irregular (Deinopis sp, Mesabolivar sp.) e orbicular (Leucauge sp e Nephila clavipes), foram mais abundantes no ambientes de centro, fato este que não foi observado na borda do fragmento, ocorrendo predominância de aranhas errantes, representadas por Wulfila sp e 5 espécies de Salticidae.

Nossos resultados demonstram que a diferença na diversidade de espécies encontradas entre os ambientes de borda e do centro do fragmento, varia de acordo com a estruturação física do ambiente, que diferiu em alguns aspectos entre os ambientes, corroborando com o proposto por Rosenzweig (1995). Este autor sugere que vários fatores ambientais afetam a diversidade de espécies, entre os quais estão incluídos: sazonalidade, heterogeneidade espacial, competição, predação, tipo de habitat, estabilidade ambiental e produtividade. Uetz (1991) afirma que a estrutura da vegetação juntamente com a heterogeneidade de hábitats são um dos fatores que exercem maior influência sobre a araneofauna, sendo determinante tanto para as aranhas construtoras de teia como para aquelas que forrageiam ativamente, pois são extremamente sensíveis a pequenas variações no habitat, incluindo a complexidade, profundidade do folhiço e microclima. (Uetz 1991, Hurd \& Fagon 1992). Para análise do efeito de borda sobre a diversidade de espécies é necessário um estudo da estrutura da vegetação associado a coletas de animais considerados bioindicadores, visto que esses elementos analisados separadamente não proporcionariam subsídios suficientes para a caracterização de uma área (Ysnel \& Canard 2000).

Ricklefs (1996) afirma que nenhum organismo pode viver em todos os tipos de habitats, manipular todos os tipos de presas, ou suportar quaisquer condições físicas igualmente bem. Assim, os ambientes heterogêneos requerem que os indivíduos façam escolhas que permitam uma otimização do uso do habitat. Este fato pode ser observado no PMP ao analisar os dados a cerca da estrutura física do mesmo, diversidade e composição das comunidades de aranhas ali presentes. A araneofauna está distribuída conforme os recursos encontrados. Os pontos de centro, situados em áreas que sofrem constantemente com a lixiviação do solo, o que inviabiliza a presença de aranhas forrageadoras, em contrapartida as áreas são menos afetadas por ações antrópicas, como desmatamento e formação de trilhas, visitação para prática de esportes e lazer, favorecendo assim a predominância de vegetação herbácea e arbustiva na área (Teles 2001), conseqüentemente mais recursos alimentares, hastes para fixação de teia ou abrigo para aranhas construtoras de teia. Em contrapartida, ao observar os pontos de borda, verifica-se uma estrutura física diferente, caracterizando o PMP como um ambiente heterogêneo, fato também observado por Cruz Rios et al. (2003) ao estudarem comunidades de anfíbios e répteis, e Teles (2001) ao estudar a flora do PMP. O CAP mais acentuado na borda, associado a maior profundidade de serrapilheira tende a disponibilizar mais recursos para forrageio e predação, tornando a borda do PMP uma área de distribuição livre ideal (Ricklefs 1996), onde cada indivíduo na população explora uma fonte de igual qualidade percebida a despeito da variação intrínseca da qualidade da parte na ausência de consumidores.

A presença de uma maior diversidade de espécies de aranhas na borda do fragmento, apresenta-se como um resultado inusitado, visto que a maioria dos trabalhos aponta maior diversidade na região de centro, devido as suas características físicas geralmente mais conservadas (Teles 
2001). Estes resultados permitem sugerir que as diferenças encontradas na diversidade de espécies entre os dois ambientes estão diretamente relacionadas à caracterização física do Parque, gerando uma dicotomia da distribuição das aranhas de acordo com a disponibilidade de recursos, associado à estrutura física do PMP, onde podemos verificar que as aranhas de teia predominam nas áreas de centro, enquanto as aranhas errantes nas áreas de borda. Desta forma evidencia-se a atuação do efeito de borda como uma barreira natural, impedindo assim o fluxo de espécies entre os ambientes.

\section{Agradecimentos}

Agradecemos ao amigo Moacir Tinoco pela redação do abstract, e por estar sempre presente nas discussões a respeito das análises estatísticas e desenhos amostrais ideais aplicados no PMP, e também a Henrique Browne por esclarecer muitas dúvidas a respeito da Bioestatística. Ao também amigo Valdo França por colaborar com os trabalhos em campo, sem ele seria muito difícil realizá-los. E ao Centro de Ecologia e Conservação Animal, Instituto de Ciências Biológicas, UCSal - ECOA/ICB/UCSal, por fornecer todo o apoio logístico necessário para a realização deste trabalho.

\section{Referências Bibliográficas}

AYRES, M., M. AYRES JR., D.L. AYRES, A.A. SANTOS. 2000. Bioestat 2.0. USP. São Paulo, SP, Brasil.

BENEDETTI, V. \& ZANI FILHO, J. 1993. Metodologia para caracterização de fragmentos florestais em projetos agrosilviculturais. In: Congresso Florestal Brasileiro. CuritibaPR. Anais. v.2. p.400-401.

BREENE, R.G., D.A. DEAN, M. NYFFELER, G.B. EDWARDS. 1993. Biology, predation an significance of spiders in texas Cotton ecosystems - with a key to the species. Departament of entomology, Texas A \& M University College Station, Texas.

CODDINGTON, J.A., LEVI, H.W. 1991. Systematics and evolution of spiders (Araneae). Ann. Rev. Ecol. Syst. 22: 565-592.

CONCEIÇÃO, A. \& PEREIRA, A. D. 1998. Flora do Parque Metropolitano de Pituaçu, Salvador-BA, BrasilGênero Stylosanthes SW. (Fabaceae) in: Resumos do XLIX Congresso Nacional de Botânica. Salvador: Universidade Federal da Bahia. p. 365.

CRUZ RIOS, R.H, RIBEIRO, H.C.B, LIMA, M.T, TINOCO, M.S. 2003. Aspectos da estrutura das comunidades de anfíbios e répteis (Vertebrata;Tetrapoda) e sua relação com a diversidade de paisagens no Parque Metropolitano de Pituaçu (PMP), Salvador, Bahia, Brasil. In : Anais de trabalhos completos do VI Congresso de Ecologia do Brasil. Fortaleza, Ceará. p.141
ESPÍRITO-SANTO, F.D.B, OLIVEIRA-FILHO, A.T. (de), MACHADO, E.L.M, SOUZA, J.S., FONTES, M.A.M.L. \& MARQUES, J.J.G.de S. 2002. Variáveis ambientais e a distribuição de espécies arbóreas em um remanescente de floresta estacional semidecídua Montana no Campo da Universidade Federal de Lavras, MG. Acta bot. bras. 16(3): 331-356.

FOELIX, R.F. 1996. Biology of spiders. Oxford University press, New York - Oxford - $2^{\mathrm{a}}$ ed.

HACKER, S.D. \& S.T. GAINES. 1997. Some implications of direct positive interactions for community species diversity. Ecology, 78(7): 1990-2003.

HENDERSON, P.A. \& SEABY, R.M.H. 1998. Diversity 2.2. Disponível em: http//:www.irchouse.demon.co.uk/ Acesso: 30/10/04.

HÖFER, H. \& BRESCOVIT,A.D. 2001. Species and guild structure of a Neotropical spider asseblage (Araneae) from Reserva Ducke, Amazonas, Brazil. Andrias, 15: 99-119.

HURD, L.E. \& FAGON, W.F. 1992. Cursorial spiders and sucession: age or habitat structure? Oecologia 92: 215-221.

LAURENCE, W. F., FERREIRA, L. V., RANKIN-DEMERONA, J.M. \& LAURENCE, S.G. 1998. Rain Florest fragmentacion and the dynamics of Amazonian tree communities. Ecology 79(6):2032-2040.

LEVEY, D.J. 1988. Tropical wet forest treefall gaps and distributions of understory birds and plants. Ecology, 69(4): 1076-1089.

MYERS, N., MITTERMEIER, R.A., MITTERMEIER,C.G., GUSTAVO A.B. DA FONSECA \& KENT J. 2000. Biodiversity hotspots for conservation priorities. Nature 403: 853-858.

ODUM, E.P. 1988. Ecologia. Editora Guanabara Koogan, Rio de Janeiro - RJ.

PLATNICK, N.I. 2004. The world spider catalog, version 4.5(online) - Disponível em: http:/research.amnh.org/entomology/spiders/catalog 81-87/COUNTS.htm. Acesso: 21/10/04.

POLIS, G.A. 1990.The biology of scorpions. Stanford University Press.

RICKLEFS, R.E. 1996. Aeconomia da natureza, $3^{\text {a }}$ Ed., Editora Guanabara Koogan, Rio de Janeiro, RJ, p. 333-337.

ROSENZWEIG, M.L. 1995. Species diversity in space and time. Cambridge University Press. New York.

SANTOS, A. J. (dos). 1999. Diversidade e composição em espécies de aranhas da Reserva Florestal da Companhia Vale do Rio Doce (LINHARES/ES). Dissertação de Mestrado da Unicamp. Campinas, SP, 109p.

TELES, A. M. \& BAUTISTA, H. P. 2001. Flora do Parque Metropolitano de Pituaçu e seus arredores, Salvador, Bahia: Compositae In: Resumos do $52^{\circ}$ Congresso Nacional de Botânica. João Pessoa: Espaço Cultural José Lins do Rego. p. 235. 
TOTI, D.S., F.A. COYLE \& J.A. MILLER. 2000. A structured inventory of appalachian grass bald and heath bald spider assemblages and a test of species richness estimator performance. J. Arachnol. 28: 329 -345.

UETZ, G.W. 1991. Habitat structure and spider foraging. In: S.S. Bell, E.D. McCoy, H.R. Mushinsky Habitat

structure: The Physical arrangement of objects in space, eds., pp. 325-348. London, Chapman and hall.

VIANA, V.M., TABANEZ, A.J.A., MARTINEZ, J.L.A. 1992. Restauração e manejo de fragmentos florestais. In: Congresso Nacional sobre Essências Nativas, 2. São Paulo-SP. P.400-406

WISE, D.H. 1993. Spiders in ecological webs. Cambridge University Press, Cambridge.

YSNEL, F. \& CANARD, A. 2000. Spider biodiversity in connection with the vegetation structure, and the foliage orientation of hedge. J. Arachnol.28: 107-114.

Título: Estudo das comunidades de aranhas (Arachnida: Araneae) em ambiente de Mata Atlântica no Parque Metropolitano de Pituaçu - PMP, Salvador, Bahia.

Autores: Alder Oliveira-Alves, Marcelo Cesar Lima Peres, Marcelo Alves Dias, Gutemberg da Silva CazaisFerreira \& Luciano Raimundo Alardo Souto

Biota Neotropica, Vol. 5 ( número 1a ): 2005

http://www.biotaneotropica.org.br/v5n1a/pt/ abstract?inventory+BN006051a2005

Recebido em 07/12/2003 - Aceito em 01/02/2005

ISSN 1676-0603

http://www.biotaneotropica.org.br 